\title{
The Research of Forest Fire Alarm System based on Video Monitoring
}

\author{
Hongyun Guan 1, a , Chuming Gong 1, b
}

School of Information Science and Technology, Donghua University, Shanghai 201620, China

ahyguan@dhu.edu.cn, bmintie_gongcm@aliyun.com

Keywords: Forest Fire, Video Monitoring, Image Processing, Infrared Image, 3G.

\begin{abstract}
In order to improve the real-time and accuracy of forest fire detection, this article has researched to the imaging characteristics of flame and smoke under visible light. According to the forest environment with complex space and various interference, this article puts forward a fire detection algorithm based on the frame difference method and hybrid Gauss background modeling method. The design of forest fire detection system based on video surveillance is given, which can realize the fire detection and alarm while in the video monitoring, it has important significance to enhance the ability of forest fire monitoring.
\end{abstract}

\section{Introduction}

Forest fire is one kind of natural disaster which is sudden and dangerous. It occurs thousands of times a year and the affected area accounts for about $0.1 \%$ of the total in the world [1]. It is difficult to estimate the damage caused by forest fire to natural resources and public safety, so early detection and timely warning is essential to the control of the fire. In the forest fire alarm system, the most critical problem is how to quickly respond to reduce the scale of the disaster. Wireless sensor networks as a solution has been widely recognized [2]. Due to the single traditional detection algorithm, the detection results will be affected. Compared with it, the main advantages of video monitoring are: wide monitoring range, high detection accuracy and more intuitive.

Therefore, the forest fire detection system based on video monitoring is proposed in this paper. Using the HD camera and infrared camera, combining the visible light and infrared band of fire detection is a great significance for improving the accuracy of forest fire detection system.

\section{System}

\subsection{The design of hardware system}

The system architecture of forest fire detection system based on video monitoring is shown in Figure 1.The system includes video capture module, PC monitoring center and alarm module.

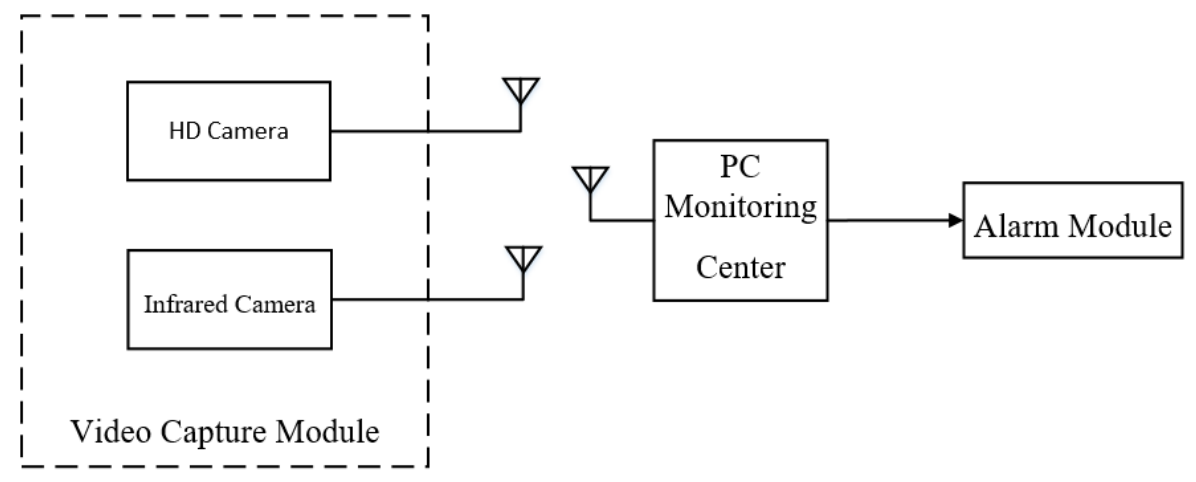

Figure 1. System structure

Firstly, video images are collected simultaneously by high definition (HD) camera and infrared camera and transmitted to the monitoring center via 3G network (PC)[3]. Secondly, the PC side carry out fire detection, respectively using fire detection algorithm in the visible and infrared at the same 
time. If the fire characteristics and the infrared imaging temperature exceeds the set threshold, judging as fire. Finally, the monitoring center alarm and GSM alarm module can accurately let the staff to understand the fire situation in the first time.

\subsection{The design of software system}

The design of software system is divided into five modules: video capture and pretreatment module, moving object detection module, fire feature detection module, infrared temperature detection module and alarm module. The flow chart of software system is shown in figure 2.

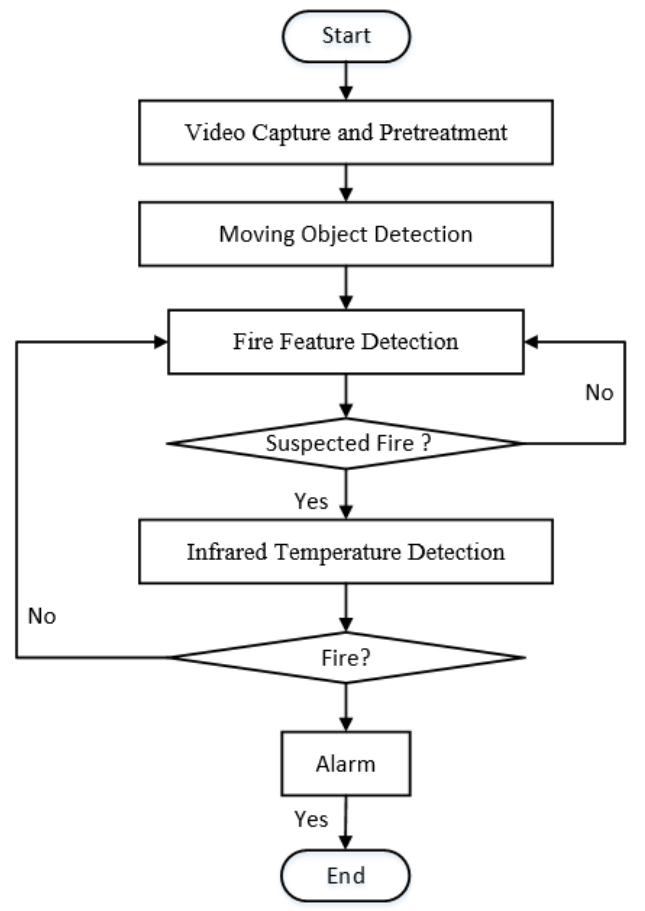

Figure 2. Flow chart of software system

This system adopts a dual judgment mode of visible light image feature recognition and infrared temperature detection, combined with multi point alarm module, which can improve the accuracy of forest fire detection system.

\section{Moving target detection}

Moving target detection methods are mainly used as optical flow method, frame difference method and background difference method etc. The change of the initial stage of smoke and flame is relatively slow and continuous, and the single detection method can't meet the complex forest environment.

\subsection{Improved frame difference method}

Frame difference method is mainly used in video sequences of continuous variation between the

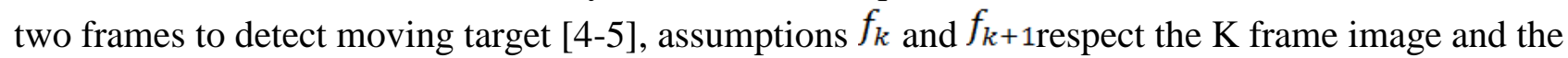
$k+1$ frame image, then the difference image of the two images as shown in equation 1.

$$
d_{k}=\left|f_{k}-f_{k+1}\right|
$$

When the image sampling interval is small, the frame difference method is not sensitive to the change of the image scene and it is easy to cause empty in the detected object. Therefore, four frame difference method is proposed based on the dynamic characteristics of the fire in this paper.

(1) Frame difference of four consecutive frames, get the two differential image binarization, as shown in equation 2 and 3.

$$
\begin{aligned}
& \mathrm{d}_{k}^{1}= \begin{cases}1, & \left|f_{k}-f_{k+2}\right|>T_{1} \\
0, & \left|f_{k}-f_{k+2}\right| \leq T_{1}\end{cases} \\
& \mathrm{d}_{k}^{2}= \begin{cases}1,\left|f_{k+1}-f_{k+3}\right|>T_{1} \\
0,\left|f_{k+1}-f_{k+3}\right| \leq T_{1}\end{cases}
\end{aligned}
$$


(2) And two binary images, the outline of the moving target intersection is obtained. As shown in equation 4.

$$
\begin{aligned}
\mathrm{D} & =\mathrm{d}_{k}^{1} \cap \mathrm{d}_{k}^{2} \\
& = \begin{cases}1, & \text { Target } \\
0, & \text { background }\end{cases}
\end{aligned}
$$

\subsection{Gaussian Mixture Model}

The core idea of Gauss mixture model is to use Gaussian model to represent the characteristics of the pixel points in the video image [6]. Forest environment, the video will contain natural light and wind and other factors, so every pixel of the background image should be modeled as a superposition of multiple Gauss distribution. It is assumed that the RGB channel of the image pixel is independent and the variance is the same, then the pixel value can be represented by the mixed Gauss model, as shown in the equation 5 .

$$
P\left(f_{t}\right)=\sum_{i=1}^{K} \frac{\omega_{i, t}}{(2 \pi)^{\frac{n}{2}}\left|\tau_{i, t}\right|^{\frac{1}{2}}} \cdot e^{-\frac{1}{2}\left(f_{t}-\mu_{i, t}\right)^{T}\left(\delta_{i, t}^{2} I\right)^{-1}\left(f_{t}-\mu_{i, t}\right)}
$$

Among them, $\mathrm{K}$ is the number of Gauss model selection; $\omega_{i, t}$ expressed as time $\mathrm{t}$ in the Gauss mixture model the I Gauss distribution function of the weights.

The Gauss mixture model has high robustness which can better adapt to the background changes and is more suitable for the detection of forest fires.

\section{Analysis}

Firstly, we use image preprocessing and motion target detection technique for the collected video the image. Then, the images are segmented by the mixed smoke color model based on RGB, HSI, HSV. With the result of space segmentation, the images of smoke are extracted finally. For the flame part, we use mixed smoke color model based on RGB, HSI, YCrCb. Figure 3 and figure 4 are the results of smoke segmentation and flame segmentation respectively. The results show that the proposed algorithm can extract the smoke and flame in the video.

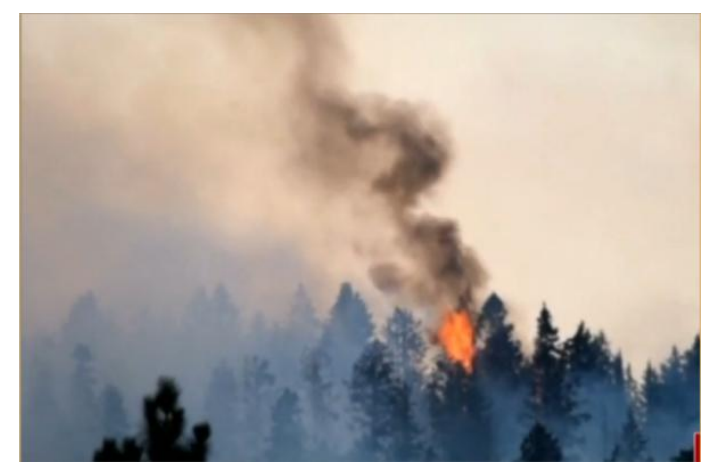

(a) Smoke video image

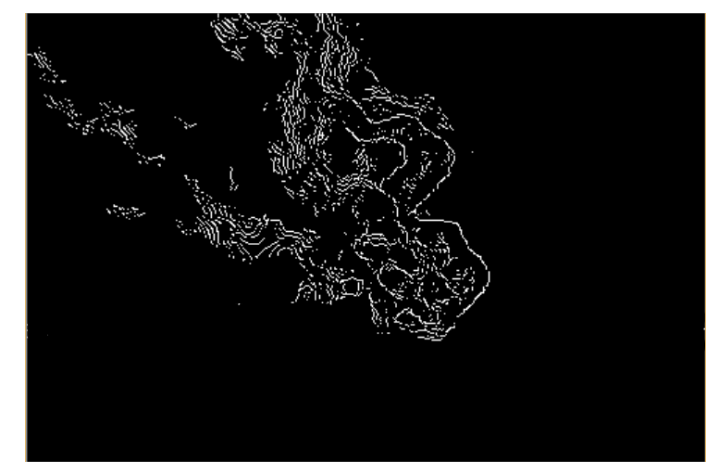

(b) Segmented smoke image

Figure 3. The segmentation result of smoke

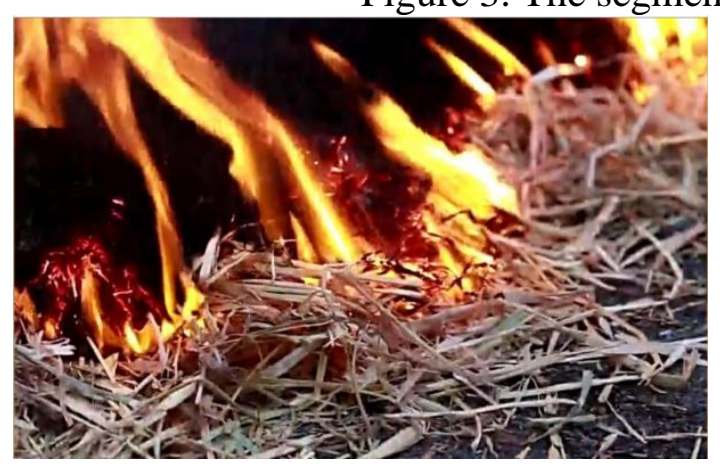

(a) Flame video image

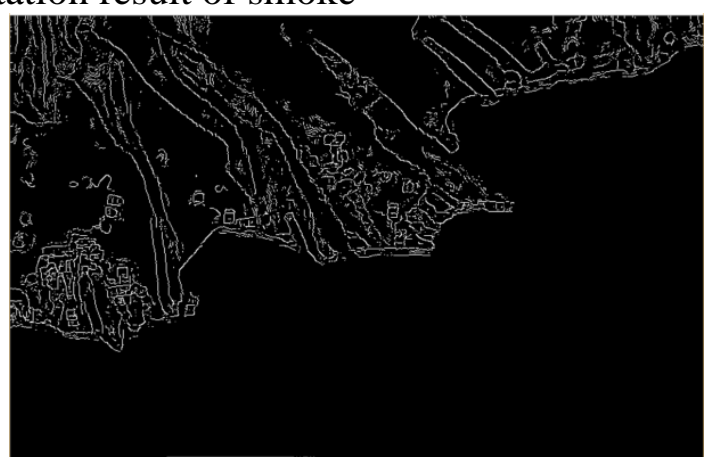

(b) Segmented flame image

Figure 4. The segmentation result of flame 
Different weather conditions and environmental conditions have a great impact on the results. Therefore, this paper selects 80 video under different conditions to test the reliability of the system. Including 40 videos with a typical smoke and flame characteristics and 40 videos with similar texture, edge characteristics. The statistical results of the tests are shown in table 1.

Table 1. The statistical results of system

\begin{tabular}{|c|c|c|}
\hline Video Type & Number of Identification/ Number of Videos & Recognition Rate \\
\hline Forest smoke & $17 / 20$ & $85 \%$ \\
\hline Smoke interference video & $3 / 20$ & $15 \%$ \\
\hline Forest flame & $18 / 20$ & $90 \%$ \\
\hline Flame interference video & $2 / 20$ & $10 \%$ \\
\hline
\end{tabular}

\section{Summary}

This paper presents a forest fire detection system based on video monitoring. Firstly, use the moving target detection algorithm based on the frame difference method and the mixed Gauss model to extract the smoke and flame moving target. Then the system is to determine whether smoke or flame in visible and infrared model. In the case of fire, start to alarm. The system can carry out real-time video fire detection and fire alarm, and achieve the desired results. Therefore, with the rapid development of wireless network technology, the application of remote video monitoring in the field of forest fire detection will have a bright future.

\section{References}

[1]. Wei Shujing, Sun Long, Wei Shuwei, et al.Effects of Climate Changes on Forest Disasters and the Preventive Measures[J].Journal of Catastrophology.Vol.28(2013)No.1,p.36-40.

[2]. Song Zhecun, Chen ning, Li Difei. Application of ZigBee Wireless Sensor Technology in Forest Conflagration Surveillance [J].Process Automation Instrumentation. Vol.32 (2011) No.4, p.50-52.

[3]. Pu Wandong, Pei Yijian. Analysis Influencing Factors of Video-based Real-time Vehicle Detection [C] 2010 International Conference on Software and Computing Technology. IEEE, 2010, p. 379-380.

[4]. Wei-Wei, Qi-Wu. Moving target detection based on three frame difference combined with improved gaussian modeling [J]. Computer Engineering and Design.2014, 35(3):949-952.

[5]. Yuan Hangcheng, Jing Wang. A Motion Image Detection Method Based on the Inter-Frame Difference Method [J]. Applied Mechanics and Materials. Vol. 490(2014) No.7, p.1283-1286.

[6]. Hong-Sun, Kai-Guo. Moving. Target detection based on the improved Gaussian mixture background model [J]. Jiangnan University Journal. Vol.13 (2014) No.3, p.269-274. 